\title{
Significant expansion and red-shifting of fluorescent protein chromophore determined through computational design and genetic code expansion
}

Li Wang ${ }^{1,2}$, Xian Chen ${ }^{3}$, Xuzhen Guo ${ }^{1,2}$, Jiasong Li ${ }^{1}$ Qi Liu', Fuying Kang ${ }^{1,2}$, Xudong Wang ${ }^{1}$, Cheng Hu ${ }^{1,2}$, Haiping Liu ${ }^{1}$, Weimin Gong ${ }^{1}$, Wei Zhuang ${ }^{4}$, Xiaohong Liu $^{1 凶}$, Jiangyun Wang ${ }^{1,2} \bowtie$

1 Institute of Biophysics, Chinese Academy of Sciences, Beijing 100101, China

2 College of Life Sciences, University of Chinese Academy of Sciences, Beijing 100049, China

3 Key Laboratory of Physics and Technology for Advanced Batteries (Ministry of Education), Department of Physics, Jilin University, Changchun 130012, China

4 State Key Laboratory of Structural Chemistry, Fujian Institute of Research on the Structure of Matter, Chinese Academy of Sciences, Fuzhou 350002, China

Received: 24 May 2018 / Accepted: 17 July 2018 / Published online: 10 November 2018

\section{Graphical abstract}

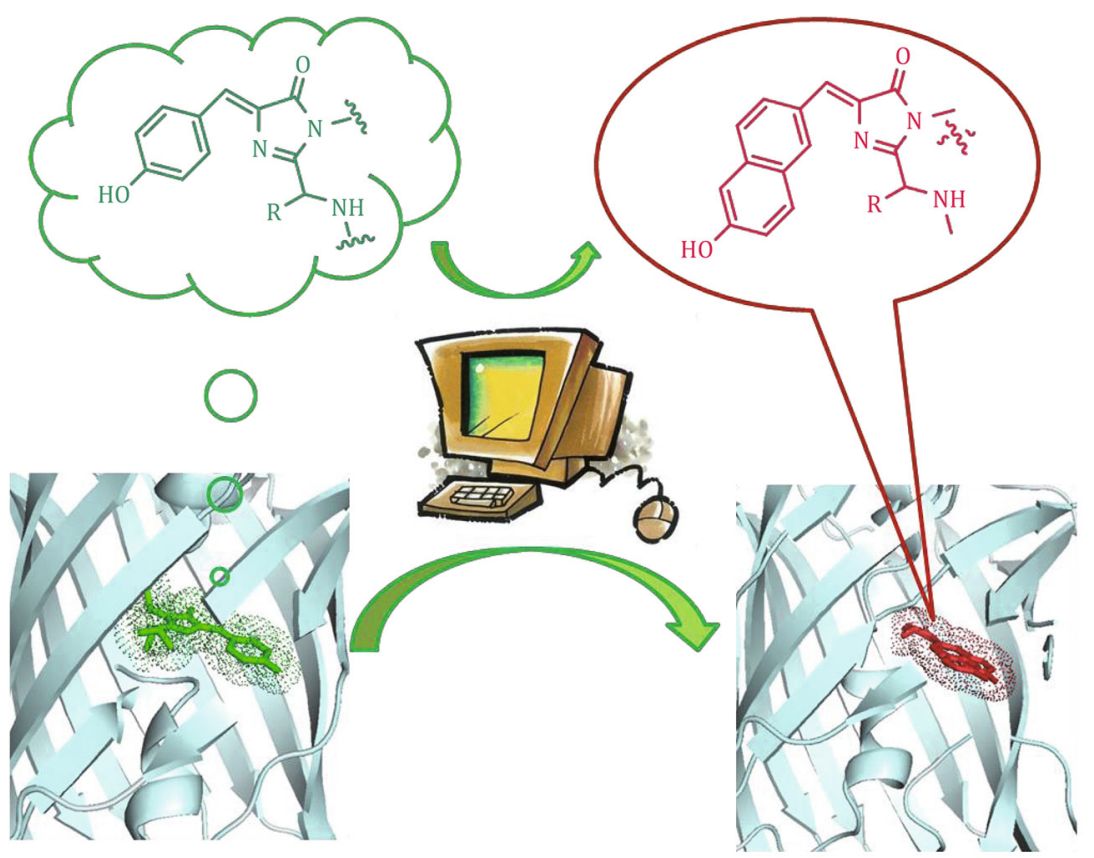

Li Wang, Xian Chen and Xuzhen Guo have contributed equally to this work.

$\bowtie$ Correspondence: wzhuang@fjirsm.ac.cn (W. Zhuang), liuxh@moon.ibp.ac.cn (X. Liu), ang@ibp.ac.cn (J. Wang) 
Abstract Fluorescent proteins (FPs) with emission wavelengths in the far-red and infrared regions of the spectrum provide powerful tools for deep-tissue and super-resolution imaging. The development of red-shifted FPs has evoked widespread interest and continuous engineering efforts. In this article, based on a computational design and genetic code expansion, we report a rational approach to significantly expand and red-shift the chromophore of green fluorescent protein (GFP). We applied computational calculations to predict the excitation and emission wavelengths of a FP chromophore harboring unnatural amino acids (UAA) and identify in silico an appropriate UAA, 2-amino-3-(6hydroxynaphthalen-2-yl)propanoic acid (naphthol-Ala). Our methodology allowed us to formulate a GFP variant (cpsfGFP-66-Naphthol-Ala) with red-shifted absorbance and emission spectral maxima exceeding 60 and $130 \mathrm{~nm}$, respectively, compared to those of GFP. The GFP chromophore is formed through autocatalytic post-translational modification to generate a planar 4-( $p$-hydroxybenzylidene)-5imidazolinone chromophore. We solved the crystal structure of cpsfGFP-66-naphthol-Ala at $1.3 \AA$ resolution and demonstrated the formation of a much larger conjugated $\pi$-system when the phenol group is replaced by naphthol. These results explain the significant red-shifting of the excitation and emission spectra of cpsfGFP-66-naphthol-Ala.

Keywords Green fluorescent protein, Red-shift, Unnatural amino acids, Computational design

\section{INTRODUCTION}

Fluorescent proteins (FPs) with far-red and infraredregion emission wavelengths are powerful tools for tracking protein localization in cells, cell migration, and deep-tissue imaging (Enterina et al. 2015; Nienhaus and Nienhaus 2014; Niu and Guo 2013; Sengupta et al. 2014; Shaner et al. 2011). Methods to achieve red-shifted FPs have evoked widespread interest and continuous engineering efforts (Chang et al. 2012; Enterina et al. 2015; Grimm et al. 2015; Newman et al. 2011; Niu and Guo 2013; Shu et al. 2009; Subach et al. 2011; Subach and Verkhusha 2012). Despite recent results, however, the design of these mutated FPs remains mostly dependent on high-throughput screening, which is a costly and tedious task. The development of modern computational chemistry allows a better understanding of the transition-state structure of FPs through theoretical calculation (Chica et al. 2010; Mou et al. 2015). Through this technology, key factors can be determined for the design of FPs with significantly red-shifted excitation and emission spectra. When the 66Tyr residue of the chromophore in various FPs is substituted with HqAla, excitation and emission spectra red-shifted by $30 \mathrm{~nm}$ are achieved (Liu et al. 2013). In the present work, a computation-based design and genetic code expansion are applied to rationally design an appropriate unnatural amino acid (UAA), 2-amino-3-(6-hydroxynaphthalen2-yl)propanoic acid (naphthol-Ala), and genetically incorporate the same to the chromophore of cpsfGFP. cpsfGFP-66-naphthol-Ala has significantly red-shifted absorbance and emission spectral maxima compared to those of wild-type green FP (GFP). Structural analysis reveals the formation of a novel naphthol-imidazolinone (NapI) chromophore with a significantly larger conjugated $\pi$ system compared to that of the original 4-( $p$-hydroxybenzylidene)-5-imidazolinone (HBI) chromophore in GFP. Furthermore, as a noteworthy and promising technology in super-resolution imaging, GFP and some of its mutants undergo efficient photoconversion into a red fluorescent state (Bogdanov et al. 2009; Elowitz et al. 1997; Saha et al. 2013). The oxidation red fluorescence of cpsfGFP-66-Naphthol-Ala revealed remarkably red-shifted excitation and emission spectra. This oxidation redding phenomenon was observed in vivo through confocal and fluorescence microscopy.

\section{RESULTS AND DISCUSSION}

\section{Calculation and rational design of red-shift FP chromophore}

When the sfGFP 66Tyr site is substituted with a heterocyclic amino acid, the resulting excitation and emission spectra are red-shifted by approximately $30 \mathrm{~nm}$ (Liu et al. 2013). The fluorescence frequencies of the red-shifted chromophores (Fig. 1, ST-1-ST-8) were first computed by the time-dependent density functional theory (TDDFT) method with the long-range-corrected (LRC) exchange-correlation functional (Laurent and Jacquemin 2013). The gas-phase vertical absorption and emission energies of the eight chromophores (ST-1-ST-8 in Fig. 1), including their neutral and anionic states, are presented in Table 1. A trend of spectral shifting trend was well represented by the calculations. From left to 
right in each row of Table 1, the absorption and emission energies of either neutral or anionic chromophores are red-shifted compared to those of their left-hand neighbors. In addition, the absorption and emission energies of neutral and anionic chromophores in lower rows are red-shifted compared to the corresponding values indicated in upper rows. All frequency-change features observed were consistent with the experiment values.

The transition dipole moments of the chromophores also revealed a regular pattern. Table 1 shows that, in general, larger transition dipole moments corresponded to more red-shifted excitation and emission spectra. The transition dipole moment of emission was consistently larger than that of absorption. Intramolecular charge transfer significantly affects the spectra of many conjugated chromophores or polymers. Population analysis indicated that, besides large intramolecular charge transfers, a free electron flow channel can also decrease the transition (absorption and emission) energy.

The calculations provide a reliable guide for the design of red-shifted FPs. First, large conjugated $\pi$ electrons afford a spacious and free electron flow place. Second, an ideal side chain should provide efficient electron-withdrawing and -donating effects. "Left-ring" type (HBI and HQI) charge transfers affect the anionic "right-ring to left-ring" charge transfer process more extensively than the neutral charge transfer process, and the neutral "left-ring to right-ring" charge transfer process is more influenced by the side chain of the "right-ring" charge transfer process than the "left-ring" transfer process. Third, in many conjugated polymer materials, $\mathrm{N}$ is a typical electron-withdrawing atom. In the case of HQI, elimination of $\mathrm{N}$ from HQI may promote the anionic "right-ring to left-ring" charge transfer process.

We predicted the structures of the heterocyclecontaining chromophores ST-9-ST-12 (Fig. 1). Using the same calculation, the fluorescence frequencies of these chromophores were computed to design more redshifted FPs. The gas-phase vertical absorption and emission energies of these chromophores (ST-9-ST-12 in Fig. 1) in their neutral and anionic states are presented in Table 1 . Compared with the absorption and<smiles>[R]C(NC(C)C)C1=N/C(=C\c2ccc(O)cc2)C(=O)N1C</smiles>

ST-1

B<smiles>[R]C(NC(C)C)C1=N/C(=C\c2ccc(O)c3ncccc23)C(=O)N1CC</smiles>

ST-5

C<smiles>[R]C(NC)C1=N/C(=C\c2ccc(O)c3ccccc23)C(=O)N1C(C)(C)C</smiles>

ST-9

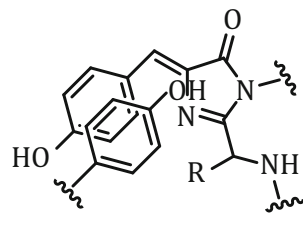

ST-2

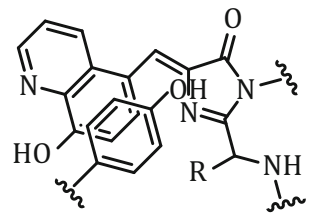

ST-6<smiles>[R]C(NC)C1=N/C(=C\c2ccc(O)c3ncncc23)C(=O)N1C(C)(C)C</smiles>

ST-10<smiles>CC1OC(C)(O)C1=NC1=NC(=Cc2ccc(O)cc2)C(=O)N1C</smiles>

ST-3<smiles>CC1OC(C)(O)N=C1C1=NC(=Cc2ccc(O)c3ncccc23)C(=O)N1C</smiles>

ST-7<smiles>[R]C(NC)C1=N/C(=C\c2ccc(O)c3[nH]cnc23)C(=O)N1C(C)(C)C</smiles>

ST-11<smiles>[R]/C(=C\C(=O)CC)C1=N/C(=C\c2ccc(O)cc2)C(=O)N1C</smiles>

ST-4<smiles>[R]/C(=C\C([CH])=O)C1=N/C(=C\c2ccc(O)c3ncccc23)C(=O)N1C</smiles>

ST-8<smiles>[R]C(NC)C1=N/C(=C\c2ccc3cc(O)ccc3c2)C(=O)N1CC</smiles>

ST-12

Fig. 1 AST-1, ST-2, ST-3, and ST-4, respectively, representing the chemical structures of sfGFP, sfYFP, PsmOrange, and eqFP650 containing the 4-( $p$-hydroxybenzylidene)-5-imidazolinone chromophore. B ST-5, ST-5, ST-7, and ST-8, respectively, representing the chemical structures of sfGFP-66-HqAla, sfYFP-66-HqAla, PsmOrange-72-HqAla, and eqFP650-67-HqAla containing the 8-hydroxyquinolinimidazolinone chromophore. C Chemical structures of the predicted chromophores ST-9-ST-12 
Table 1 Calculated absorption and emission energies (eV) of chromophore models ST-1-ST-8 and their corresponding dipole moments (a.u.)

\begin{tabular}{|c|c|c|c|c|c|c|c|c|c|}
\hline & \multicolumn{3}{|l|}{ ST-1 } & \multicolumn{2}{|l|}{ ST-2 } & \multicolumn{2}{|l|}{ ST-3 } & \multicolumn{2}{|l|}{ ST-4 } \\
\hline & $E(\Delta E)$ & Dip. & & $E(\Delta E)$ & Dip. & $E(\Delta E)$ & Dip. & $E(\Delta E)$ & Dip. \\
\hline \multicolumn{10}{|l|}{ Neutral } \\
\hline$S_{0} \rightarrow S_{1}$ & 3.87 & \multicolumn{2}{|l|}{8.04} & $3.78(-0.09)$ & 6.60 & $3.50(-0.37)$ & 9.59 & $3.16(-0.71)$ & 10.47 \\
\hline$S_{1} \rightarrow S_{0}$ & 3.27 & \multicolumn{2}{|l|}{9.28} & $3.16(-0.11)$ & 8.01 & $2.93(-0.34)$ & 11.49 & $2.69(-0.58)$ & 12.98 \\
\hline \multicolumn{10}{|l|}{ Anionic } \\
\hline$S_{0} \rightarrow S_{1}$ & 3.23 & \multicolumn{2}{|c|}{12.84} & $3.16(-0.07)$ & 11.08 & $2.75(-0.48)$ & 15.03 & $2.56(-0.67)$ & 18.41 \\
\hline$S_{1} \rightarrow S_{0}$ & 3.04 & \multicolumn{2}{|l|}{12.76} & $2.95(-0.09)$ & 10.67 & $2.59(-0.45)$ & 15.41 & $2.43(-0.61)$ & 18.80 \\
\hline \multirow[t]{4}{*}{ Exp. } & & & & \multicolumn{2}{|l|}{ Abs. $(-0.15)$} & \multicolumn{2}{|l|}{ Abs. $(-0.29)$} & \multicolumn{2}{|l|}{ Abs. $(-0.46)$} \\
\hline & & & & \multicolumn{2}{|l|}{ Emi. $(-0.08)$} & \multicolumn{2}{|l|}{ Emi. $(-0.24)$} & \multicolumn{2}{|l|}{ Emi. $(-0.52)$} \\
\hline & \multicolumn{3}{|l|}{ ST-5 } & \multicolumn{2}{|l|}{ ST-6 } & \multicolumn{2}{|l|}{ ST-7 } & \multicolumn{2}{|l|}{ ST-8 } \\
\hline & $E(\Delta E)$ & & Dip. & $E(\Delta E)$ & Dip. & $E(\Delta E)$ & Dip. & $E(\Delta E)$ & Dip. \\
\hline \multicolumn{10}{|l|}{ Neutral } \\
\hline$S_{0} \rightarrow S_{1}$ & $3.55(-0.32)$ & & 8.12 & $3.50(-0.37)$ & 6.62 & $3.21(-0.66)$ & 10.41 & $3.00(-0.87)$ & 11.36 \\
\hline$S_{1} \rightarrow S_{0}$ & $3.03(-0.24)$ & & 9.75 & $2.94(-0.33)$ & 8.08 & $2.72(-0.55)$ & 12.80 & $2.49(-0.78)$ & 14.42 \\
\hline \multicolumn{10}{|l|}{ Anionic } \\
\hline$S_{0} \rightarrow S_{1}$ & $3.12(-0.11)$ & & 11.67 & $3.01(-0.22)$ & 9.12 & $2.67(-0.56)$ & 16.12 & $2.46(-0.77)$ & 19.45 \\
\hline$S_{1} \rightarrow S_{0}$ & $2.94(-0.10)$ & & 11.19 & $2.83(-0.21)$ & 9.17 & $2.52(-0.52)$ & 16.75 & $2.34(-0.70)$ & 20.07 \\
\hline Exp. & Abs. $(-0.25)$ & & & Abs. $(-0.28)$ & & Abs. $(-0.42)$ & & Abs. $(-0.56)$ & \\
\hline & Emi. $(-0.15)$ & & & Emi. $(-0.19)$ & & Emi. $(-0.34)$ & & Emi. $(-0.61)$ & \\
\hline
\end{tabular}

" $\Delta E$ " represents the energy red-shift of models ST-2-ST-8 compared to that of the ST-1 model. The experimental absorption and emission energy red-shifts of these models relative to those of the ST-1 model are also shown

emission energies of the ST-1 chromophore in Table 2, those of the anionic ST-12 chromophore showed larger red-shifts.

\section{Synthesis and genetic incorporation of 2-amino- 3-(6-hydroxynaphthalen-2-yl) propanoic acid (hereafter termed naphthol-Ala)}

We synthesized the UAA naphthol-Ala from the best candidate chromophore (ST-12) and genetically encoded it into cpsfGFP. Naphthol-Ala can be synthesized through six steps (Fig. 2A). In brief, 6-hydroxy-2naphthoic acid is first methylated to yield methyl ester and then reduced to obtain a hydroxyl group through halogenation by sulfoxide chloride. The corresponding chloride is reacted with diethyl acetamidomalonate in alkaline conditions to form the ester precursor of the target UAA, which is then deprotected to form the hydrobromide salt of naphthol-Ala.

To deliver naphthol-Ala to the defined protein sites in E. coli through the TAG codon, a mutant $M$. jannaschii tyrosyl amber suppressor tRNA (MjtRNA $A_{\text {CUA }}^{\text {Tyr }}$ )/tyrosyltRNA synthetase (MjTyrRS) pair must be constructed. The desired MjTyrRS clone was obtained after three rounds of positive selection and two rounds of negative selection from the MjTyrRS library. This clone can resist $120 \mu \mathrm{g} / \mathrm{mL}$ chloramphenicol due to the expression of the chloramphenicol acetyl transferase (CAT) gene in the presence of $1 \mathrm{mmol} / \mathrm{L}$ naphthol-Ala but can only resist $20 \mu \mathrm{g} / \mathrm{mL}$ chloramphenicol in the absence of the UAA. The desired UAA RS was named naphthol-Ala RS. Sequencing of this clone revealed the following mutations (Table 3): Y32R, L65H, H70G, F108N, Q109C, D158N, and L162S. The Y32R and H70G mutations create additional space to accommodate the bulky naphthol group. L65, F108, and D158 are mutated to hydrophilic amino acids, thus creating a large binding pocket while providing additional hydrogen bonding interactions to stabilize the naphthol ring. Naphthol-Ala can also be incorporated into proteins with the following mutants (Chen and Tsao 2013): Y32E, L65T, D158S, I159A, H160P, Y161T, L162Q, A167W, and D286R in NpOH-RS1; and Y32E, L65V, K90E, I159A, H160W, Y161G, L162Q, A167I, and D286R in NpOH-RS2. The yield of the Z-domain mutant with NpOH-RS1 was roughly $7 \mathrm{mg} / \mathrm{L}$ culture in the presence of naphthol-Ala. These results demonstrate that the active site of tRNA $\left(M j\right.$ tRNA $\left.A_{\text {CUA }}^{\mathrm{Tyr}}\right) /$ tyrosyl-tRNA synthetase has high capacity 
Table 2 Calculated absorption and emission energies $(\mathrm{eV})$ of the chromophore models ST-9-ST12

“ $\Delta E$ " refers to the energy redshift of the four predicted chromophore models compared to that of the ST- 1 chromophore model

\begin{tabular}{lllll}
\hline & ST-9 & ST-10 & ST-11 & ST-12 \\
& $E(\Delta E)$ & $E(\Delta E)$ & $E(\Delta E)$ & $E(\Delta E)$ \\
\hline $\begin{array}{l}\text { Neutral } \\
\quad\end{array}$ & & & \\
$S_{0} \rightarrow S_{1}$ & $3.51(-0.36)$ & $3.56(-0.31)$ & $3.72(-0.15)$ & $3.60(-0.27)$ \\
$S_{1} \rightarrow S_{0}$ & $3.03(-0.24)$ & $3.00(-0.27)$ & $3.19(-0.08)$ & $3.08(-0.19)$ \\
Anionic & & & & \\
$S_{0} \rightarrow S_{1}$ & $3.09(-0.14)$ & $3.06(-0.17)$ & $3.21(-0.02)$ & $2.72(-0.51)$ \\
$S_{1} \rightarrow S_{0}$ & $2.94(-0.10)$ & $2.58(-0.46)$ & $2.97(-0.07)$ & $2.56(-0.48)$ \\
Exp. & & & & Abs. $(-0.27)$ \\
& & & Emi. $(-0.49)$ \\
\hline
\end{tabular}

A

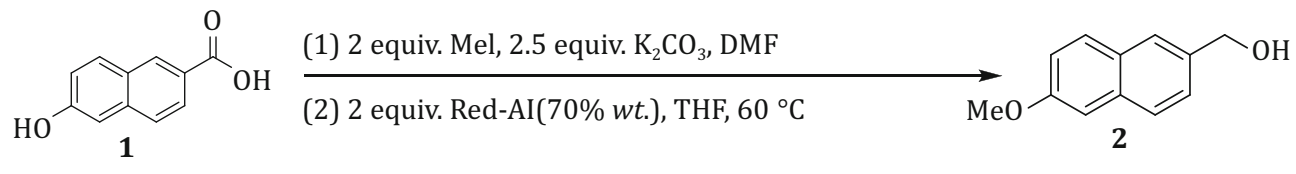

(1) 3 equiv. $\mathrm{SOCl}_{2}$, DCM, ice bath

(2) 1.5 equiv. Diethyl acetamidomlonate 3 equiv. $\mathrm{NaH}, \mathrm{DMF}$

COOEt<smiles>CCOC(=O)C(C)(N)Cc1ccc2cc(OC)ccc2c1</smiles>

(1) $\operatorname{HBr}(48 \% w / v$, aq), reflux

(2) $\mathrm{NaOH}(5 \mathrm{~mol} / \mathrm{L}, \mathrm{aq})$, adjust to $\mathrm{pH}=6-7$<smiles>NC(Cc1ccc2cc(O)ccc2c1)C(=O)O</smiles>

B

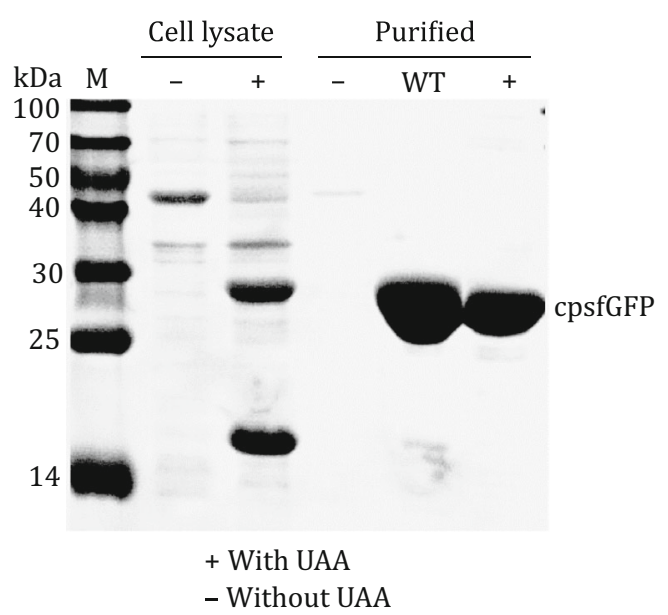

C

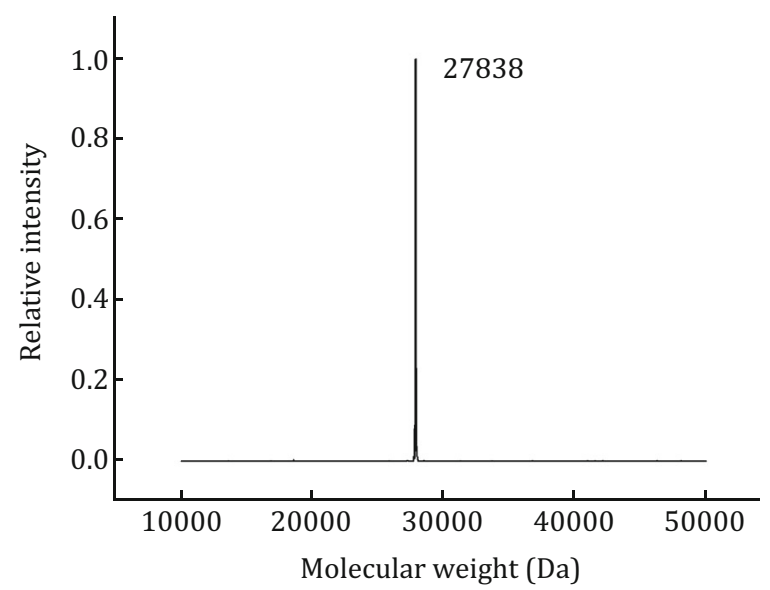

Fig. 2 A Synthesis of 2-amino-3-(6-hydroxynaphthalen-2-yl) propanoic acid (naphthol-Ala). B Coomassie-stained SDS-PAGE gel indicating the expression of the cpsfGFP-66-naphthol-Ala mutant in the presence and absence of $1 \mathrm{mmol} / \mathrm{L}$ naphthol-Ala. C ESI-MS spectra of the TAG66 mutant of cpsfGFP, expected mass: 27873 Da, found: 27873.27 Da

and variability for incorporating even the same UAA. To enhance the yield of the mutant proteins, naphthol-Ala RS was constructed on the pEVOL system.
To determine the efficiency and fidelity of naphtholAla incorporation, an amber stop code was substituted for the Tyr66 site in cpsfGFP containing a C-terminal 
His6 tag. Protein expression was carried out in E. coli in the presence of the selected synthetase (naphthol-Ala RS), $M j$ tRNA CUA $_{\text {Ty }}^{\text {with }} 0.2 \%$ arabinose, $1 \mathrm{mmol} / \mathrm{L}$ IPTG, and $1 \mathrm{mmol} / \mathrm{L}$ UAA. The protein expressed in the absence of the UAA was taken as the negative control. Analysis of the cell lysate and purified protein by Coomassie-stained SDS-PAGE showed that the fulllength cpsfGFP mutant is expressed efficiently only in the presence of naphthol-Ala, thereby indicating the specific activity of naphthol-Ala RS for naphthol-Ala only (Fig. 2B). The yield of the Tyr66 mutant cpsfGFP (termed cpsfGFP-66-Naphthol-Ala) was $30 \mathrm{mg} / \mathrm{L}$, whereas that of sfGFP was $100 \mathrm{mg} / \mathrm{L}$. ESI-MS revealed that cpsfGFP-66-naphthol-Ala has an average mass of 27,873.27 Da (Fig. 2C), consistent with the calculated mass of 27,873 Da for the Y66 $\rightarrow$ naphthol-Ala cpsfGFP mutant, where the chromophore of the cpsfGFP mutant is fully matured after self-catalyzed dehydration and oxidation.

\section{Fluorescence characterization of cpsfGFP-66- naphthol-Ala}

We characterized the fluorescent property of cpsfGFP66-naphtol-Ala, which shows red-shifted anionic state absorbance and emission spectral maxima at 545 and $640 \mathrm{~nm}$, respectively (Fig. 3A, Table 4); these values are higher than those of sfGFP by 60 and $130 \mathrm{~nm}$, respectively. The excitation and emission spectra of naphtholAla do not show any significant overlap with those of cpsfGFP-66-naphthol-Ala (Fig. 3B), thus indicating that the observed red-shift is not due to UAA small molecules.

We performed UV-Vis titrations of cpsfGFP-66naphthol-Ala at various $\mathrm{pH}$. Figure $4 \mathrm{~A}$ shows that neutral cpsfGFP-66-naphthol-Ala displays a strong absorption peak at $450 \mathrm{~nm}$ and that new peaks corresponding to the anionic state appear at $545 \mathrm{~nm}$ with the increasing $\mathrm{pH}(\mathrm{pKa}=7.7)$. The CD spectra of cpsfGFP66-naphthol-Ala from pH 5-10 show that the secondary $\beta$-sheet structure does not change with $\mathrm{pH}$ fluctuations, thereby indicating that the protein remains correctly folded throughout $\mathrm{pH}$ titration (Fig. 4B). These results are consistent with the results predicted by the computational calculations. The detected emission red-shift energy $(-0.49 \mathrm{eV})$ of cpsfGFP-66-naphthol-Ala is comparable with the calculated emission red-shift energy of the ST- 12 chromophore, which has a $\Delta E$ value of $-0.47 \mathrm{eV}$ (Table 2). The CD spectra of cpsfGFP-66naphthol-Ala in the presence of buffers of various $\mathrm{pH}$ show no difference. (Fig 4C),

As potential tools in super-resolution imaging for investigating dynamic processes in living cells, GFP and some of its mutants undergo efficient photoconversion to a red fluorescent state. Under anaerobic conditions, the redding state of GFP shows excitation-emission maxima at 525 and $600 \mathrm{~nm}$, respectively. In the presence of potassium ferricyanide, EGFP shows red-shifted fluorescence spectra with excitation and emission peaks at 575 and $607 \mathrm{~nm}$, respectively (Bogdanov et al. 2009). We thus investigated whether cpsfGFP-66-naphthol-Ala can facilitate the oxidation redding process in the presence of potassium ferricyanide. After incorporating naphthol-Ala into the 66th position of cpsfGFP and irradiating the mutant protein using a $450-\mathrm{nm}$ laser $\left(100 \mathrm{~mW} / \mathrm{cm}^{2}\right)$ for $2 \mathrm{~min}$ in the presence of $1 \mathrm{mmol} / \mathrm{L}$ potassium ferricyanide, cpsfGFP-66-naphthol-Ala showed a remarkable red-shift with excitation and emission spectral maxima at 620 and $695 \mathrm{~nm}$, respectively (Fig. 3A). These results indicate that oxidation redding can also occur in the presence of cpsfGFP-66naphthol-Ala. Although the mechanism of GFP redding remains under investigation, it likely involves a twoelectron oxidation process, where the amino acids near the chromophore, such as Glu222 (Saha et al. 2013), may act as an electron donor, and potassium ferricyanide acts as an electron acceptor. Upon electron transfer between these groups, a Ds-red-like chromophore is formed.

\section{Crystallography characterization of cpsfGFP-66- naphthol-Ala}

To elucidate structural changes causing the red-shifted absorption and emission spectra of cpsfGFP-66naphthol-Ala, we determined the structure of cpsfGFP66-naphthol-Ala at $1.3 \AA$ Å resolution. Figure 5 shows that substitution of Tyr66 by naphthol-Ala causes substantial crowding around the chromophore. The protein backbone of cpsfGFP-66-naphthol-Ala is highly similar

Table 3 Sequence of naphthol-Ala-specific aaRSs

\begin{tabular}{llllllllll}
\hline Position & 32 & 65 & 70 & 108 & 109 & 158 & 162 & 164 & 200 \\
\hline Tyr RS & Y & L & H & F & Q & D & L & Y & D \\
Naphthol-Ala RS(8) & R & Y & G & N & C & N & S & \\
\hline
\end{tabular}


A

- cpsfGFP-66-Naphthol-Ala Ex $=440 \mathrm{~nm}$

- cpsfGFP-66-Naphthol-Ala Em $=640 \mathrm{~nm}$

- cpsfGFP-66-Naphthol-Ala with hv Ex $=620 \mathrm{~nm}$

cpsfGFP-66-Naphthol-Ala with hv Em $=700 \mathrm{~nm}$

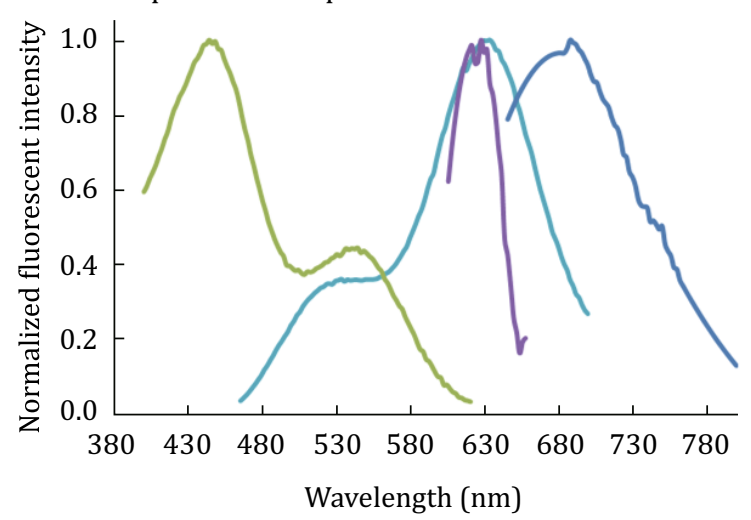

B

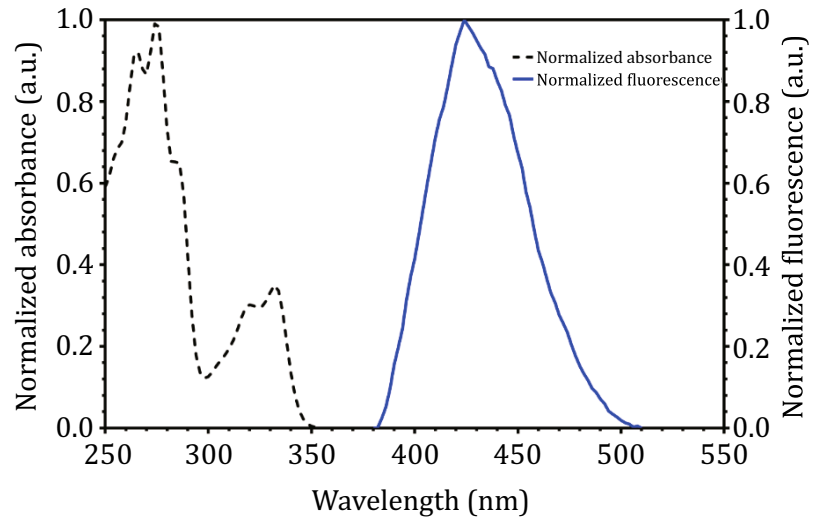

Fig. 3 A Absorption and emission spectra of cpsfGFP-66-naphthol-Ala in 20 mmol/L MOPS-citrate buffer at pH 5 before and after $450-\mathrm{nm}$ laser irradiation for $2 \mathrm{~min}$ in the presence of $1 \mathrm{mmol} / \mathrm{L}$ potassium ferricyanide. B Normalized absorption and emission spectra of $10 \mathrm{mmol} / \mathrm{L}$ naphthol-Ala in $60 \mathrm{mmol} / \mathrm{L}$ Tris-HCl buffer at $\mathrm{pH}$ 7, Ex,max $=331 \mathrm{~nm}$; Em,max $=424 \mathrm{~nm}$

Table 4 Summary of the key characteristics of fluorescent proteins

\begin{tabular}{|c|c|c|c|c|}
\hline \multirow[b]{2}{*}{ Excitation peak (nm) } & \multicolumn{2}{|c|}{ cpsfGFP-66-naphthol-Ala } & \multirow{2}{*}{$\begin{array}{l}\text { cpsfGFP-66-naphthol-Ala } \\
\text { after } 405-n m \text { irradation } \\
620 \mathrm{~nm}\end{array}$} & \multirow{2}{*}{$\begin{array}{l}\text { eqFP670 } \\
605\end{array}$} \\
\hline & 450 & 545 & & \\
\hline Emission peak (nm) & 637 & 640 & $695 \mathrm{~nm}$ & 670 \\
\hline Fluorescence QY & 0.15 & 0.02 & - & 0.06 \\
\hline $\begin{array}{l}\text { Molar extinction coefficient }(\mathrm{l} /(\mathrm{mol} \cdot \mathrm{cm})) \\
\text { at excitation maximum }(\mathrm{pH} 7.4)\end{array}$ & 82,600 & 12,700 & - & 70,000 \\
\hline Brightness $^{\text {a }}$ (a.u.) & 12,390 & 254 & - & 4200 \\
\hline Photostability, confocal $^{\mathrm{b}}(\mathrm{s})$ & - & $7.5^{\mathrm{c}}$ & - & 75 \\
\hline $\mathrm{p} K \mathrm{a}$ & 7.7 & 7.7 & - & 4.5 \\
\hline Reference & This work & This work & This work & Shcherbo et al. (2010) \\
\hline
\end{tabular}

${ }^{\mathrm{a}}$ Calculated as the product of the molar extinction coefficient and quantum yield

${ }^{\mathrm{b}}$ Time to bleach $50 \%$ of the brightness of the fluorescence signal

${ }^{\mathrm{c}}$ Excitation wavelength is $561 \mathrm{~nm}$

to that of sfGFP. The 66th naphthol-Ala group presents two conformations due to the higher accessibility of the chromophore to solvents compared with sfGFP. The naphthol and imidazolinone rings are co-planar, thereby indicating that, similar to GFP, the chromophore is fully matured after nucleophilic attack of Gly67-N $\alpha$ on the carbonyl group of Ser65, followed by dehydration and oxidation of the $\alpha-\beta$ bond in the naphthol-Ala group (Chudakov et al. 2010).

\section{Confocal and fluorescence microscopy of the oxidative redding state of cpsfGFP-66- naphthol-Ala}

We tested whether the oxidative redding phenomenon of cpsfGFP-66-naphthol-Ala could be visualized through confocal and fluorescence microscopy in vivo. Before irradiation, a thin $(\sim 10 \mu \mathrm{m})$ layer of $E$. coli cells expressed with cpsfGFP-66-naphthol-Ala in $20 \mathrm{mmol} / \mathrm{L}$ 

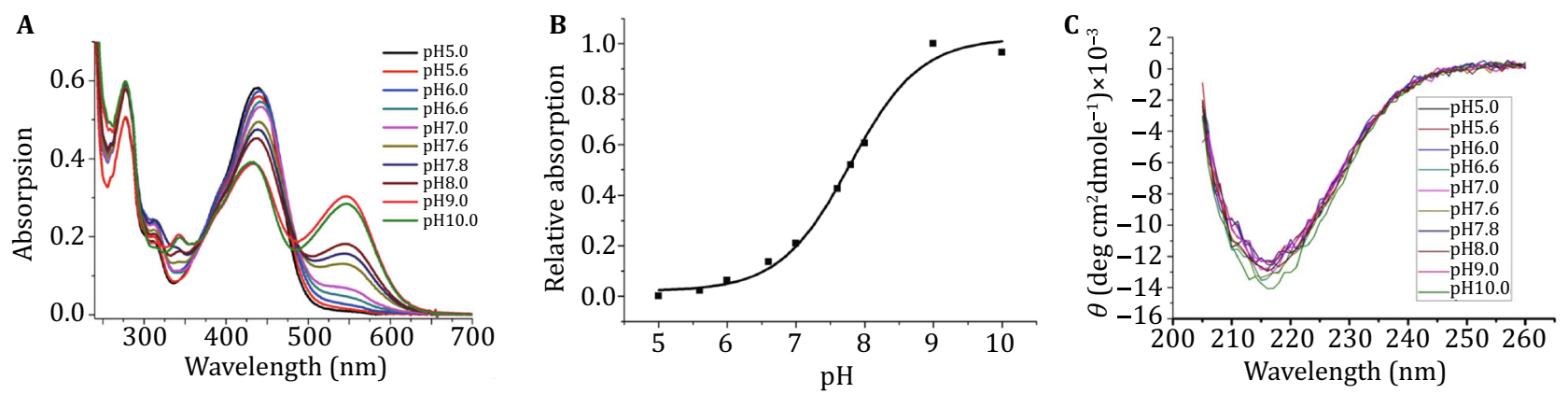

Fig. 4 A UV-Vis spectra of cpsfGFP-66-naphthol-Ala in different pH value buffer. B Anionic chromophore fraction of cpsfGFP-66naphthol-Ala at varying $\mathrm{pH}$. C CD spectra of cpsfGFP-66-naphthol-Ala in the presence of buffers of various $\mathrm{pH}$

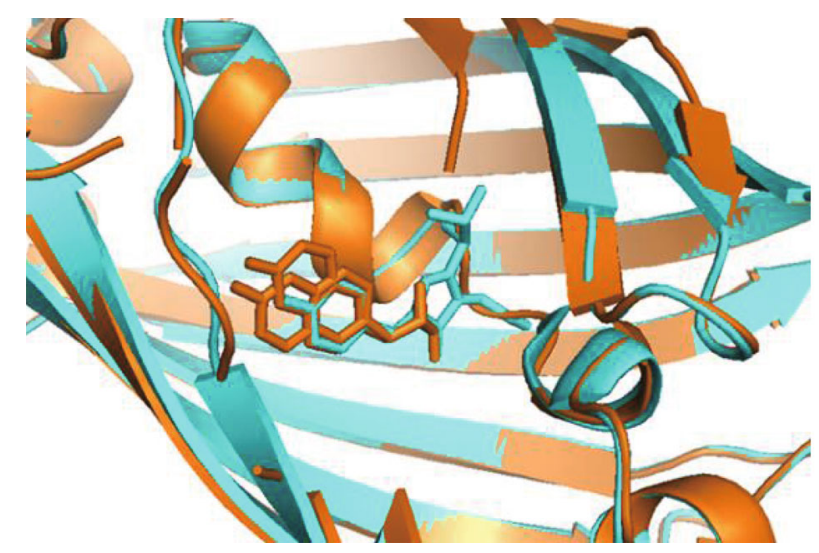

Fig. 5 Alignment of sfGFP and cpsfGFP-66-naphthol-Ala. The chromophores of cpsfGFP-66-naphthol-Ala (orange) and sfGFP (blue) show that the naphthol or phenol and imidazolinone rings are clearly coplanar

MOPS-citrate buffer at $\mathrm{pH} 5$ was prepared. The cells showed fluorescence at the detection window of 580-645 nm but were only slightly detectable at 655-755 nm. After irradiation with a 405-nm excitation laser for $2 \mathrm{~min}$ in the presence of $1 \mathrm{mmol} / \mathrm{L}$ potassium ferricyanide (highlighted by circles in Fig. 6), the fluorescence in the detection range of $655-755 \mathrm{~nm}$ increased. Overall, the oxidation redding phenomenon of cpsfGFP-66-naphthol-Ala could be observed in vivo through confocal and fluorescence microscopy.

\section{CONCLUSION}

In conclusion, we demonstrated that a red-shifted FP could be designed via a rational computational design and genetic code expansion. Our work establishes a platform for further optimization of FPs with superior properties for deep-tissue and super-resolution

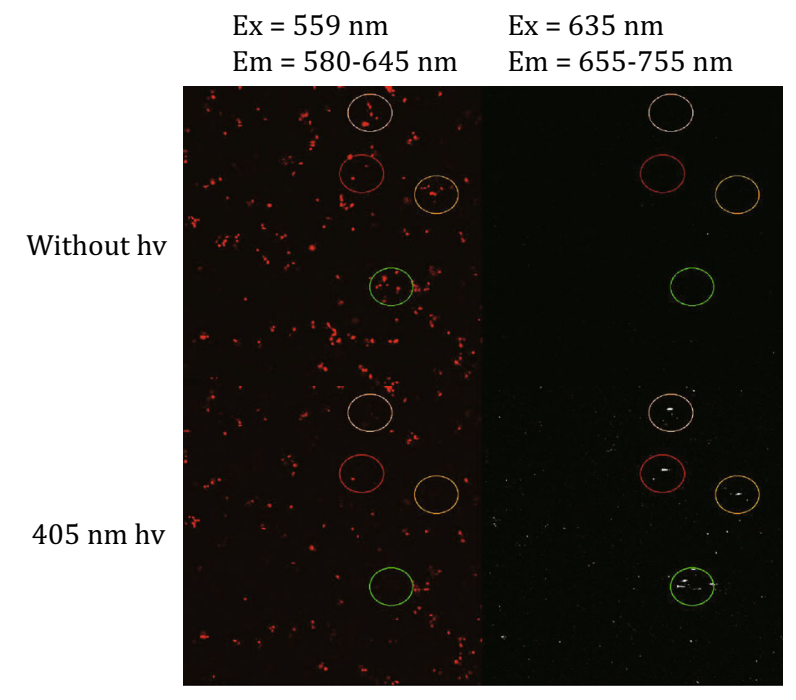

Fig. 6 Pre- and post-photoconversion images of a thin $(\sim 10 \mu \mathrm{m})$ layer of $E$. coli cells expressing cpsfGFP-66-naphthol-Ala in $20 \mathrm{mmol} / \mathrm{L}$ MOPS-citrate buffer at pH 5 under 405-nm excitation delivered through a 1.2-NA objective to the region within the circle in bleaching mode (Red channel: 580-645 nm; Red channel: $655-755 \mathrm{~nm}$ ). Photoconversion was performed at $37 \%$ laser power and 30 iterations for $111 \mathrm{~ms}$

imaging. The method presented in this work will benefit further studies on FP engineering.

\section{EXPERIMENTAL SECTION}

\section{Materials and reagents}

6-Hydroxy-2-naphthoic acid was purchased from J\&K chemical. All other chemicals used in this work were purchased from Sigma-Aldrich and applied without further purification. Silica gel chromatography purification was carried out using Silica Gel 60 (230-400 mesh). The PCR reagents, T4 DNA ligase, and restriction endonucleases were purchased from 
Fermentas. The Ni-resin (NTA) affinity purification reagents and column were purchased from Qiagen. Genes and primers were synthesized by Sangon Biotech.

\section{Instrument}

All ${ }^{1} \mathrm{H}$-NMR spectra are reported in parts per million (ppm) and were measured relative to the signals of DMSO (2.5 ppm). ${ }^{13} \mathrm{C}$-NMR spectra are reported in ppm relative to those of residual DMSO (40 ppm). The mass spectra of the chemicals were obtained using a Waters LC-MS instrument equipped with a single-quadrupole mass detector and an electrospray ionization source (Waters ACQUITY QDa), while those of the proteins were obtained using Agilent 6100 equipment with a series of triple-quadrupole mass spectrometers (Agilent Technologies, CA, USA). Protein purification was performed using AKTA UPC 900 FPLC system (GE Healthcare), and absorption spectra were obtained at room temperature using a UV-visible spectrometer (Agilent 8453, Agilent Technologies). Fluorescence spectra were obtained using a microplate reader equipped with SkanIt 2.4.3 RE software for Varioskan Flash (Varioskan Flash, Thermo Fisher Scientific Inc.). Cell fluorescent images were recorded at room temperature by confocal and fluorescence microscopy (FV1000, OLYMPUS).

\section{Theoretical calculation}

The chromophores used for the calculations were taken from a structural model (PDB: 4JFG) and built with manual modifications. The excited-state geometry of each chromophore was calculated using the TDDFT and LRC methods. The TDDFT method greatly reduces computational costs compared with MCSCF techniques, while the LRC method can deal with long-range electron correlations, which are common in $\pi$-conjugated fluorescent chromophore transitions, with greater ease than conventional hybrid functionals, such as B3LYP. In Filippi's work, the LRC functionals CAM-B3LYP and LCBLYP presented overall good agreement with the extrapolated results of solution experiments in vacuum conditions for wild-type GFP and wave function methods. All calculations were performed using the Gaussian 09 suite of programs (Frisch et al. 2016).

\section{Synthesis of (6-methoxynaphthalen-2- yl)methanol (2)}

6-Hydroxy-2-naphthoic acid (1) (3.764 g, $20 \mathrm{mmol}$ ) was dissolved in anhydrous DMF (30 ml), after which $\mathrm{K}_{2} \mathrm{CO}_{3}$ $(6.91 \mathrm{~g}, 50 \mathrm{mmol}, 2.5$ equiv.) and $\mathrm{MeI}(2.80 \mathrm{ml}$, $45 \mathrm{mmol}, 2.25$ equiv.) were added to their mixture. The reaction mixture was stirred vigorously for $48 \mathrm{~h}$ at room temperature, diluted with ice-water $(300 \mathrm{ml})$, and extracted with ethyl acetate $(3 \times 100 \mathrm{ml})$. The organic phase was washed with brine and dried with $\mathrm{Na}_{2} \mathrm{SO}_{4}$, and the solvent was evaporated under reduced pressure to yield an off-white solid precipitate. This solid was dissolved in anhydrous THF $(50 \mathrm{ml})$, after which a solution of sodium bis(2-methoxyethoxy) aluminum hydride (Red-Al, $70 w t \%)$ in toluene $(14.6 \mathrm{ml}, 40 \mathrm{mmol}$, 2 equiv.) was added dropwise to it under a nitrogen atmosphere. The mixture was refluxed at $60{ }^{\circ} \mathrm{C}$ for $4 \mathrm{~h}$ and then quenched with water $(2.5 \mathrm{ml})$. The supernatant was obtained by centrifugation, and the solvent was removed under reduced pressure. Flash column chromatography (petrol ether/ethyl acetate 1:1) formed compound 2 (3.61 g, $19.2 \mathrm{mmol}, 96 \%$ ) as a white solid.

\section{Synthesis of diethyl 2-acetamido-2-((6- methoxynaphthalen-2-yl)methyl) malonate (3)}

Compound 2 (3.61 g, $19.2 \mathrm{mmol}$ ) was dissolved in anhydrous dichloromethane $(30 \mathrm{ml})$ and then cooled in an ice-bath. Sulfoxide chloride $(4.4 \mathrm{ml}, 60 \mathrm{mmol}$, 3 equiv.) was added to the solution dropwise, and the mixture was stirred at $0{ }^{\circ} \mathrm{C}$ for $2 \mathrm{~h}$. The solvent was removed, and the residue was co-evaporated twice with dichloromethane $(20 \mathrm{ml})$ to yield a faint yellow solid.

A suspension of diethyl acetamidomalonate $(6.26 \mathrm{~g}$, $28.8 \mathrm{mmol}, 1.5$ equiv.) in anhydrous DMF (30 ml) was added to sodium hydride ( $1.38 \mathrm{~g}, 57.5 \mathrm{mmol}, 3$ equiv.) in small portions, and the mixture was stirred for 30 min under ice bath. Menaphthyl chloride dissolved in anhydrous DMF (30 $\mathrm{ml}$ ) was then added dropwise to this solution. The mixture was stirred overnight at room temperature, diluted with ice-water $(200 \mathrm{ml})$, and extracted with ethyl acetate $(200 \mathrm{ml})$. The organic phase was washed with brine and dried with $\mathrm{Na}_{2} \mathrm{SO}_{4}$, and the solvent was removed under reduced pressure. The residue was purified by flash column chromatography (petrol ether/ethyl acetate 1:1) to yield compound 3 as a white solid (6.74 g, $17.4 \mathrm{mmol}, 90.6 \%$ ).

\section{Synthesis of 2-amino-3-(6-hydroxynaphthalen-2- yl) propanoic acid hydrobromide (naphthol- Ala $\cdot \mathrm{HBr}$ )}

Compound 3 (6.74 g, $17.4 \mathrm{mmol})$ was dissolved in hydrogen bromide $(40 \mathrm{ml}, 48 \% w / v$, aq. $)$ and then refluxed at $110{ }^{\circ} \mathrm{C}$ overnight under a nitrogen atmosphere. The red solution was cooled to room temperature and then neutralized with $5 \mathrm{~mol} / \mathrm{L} \mathrm{NaOH}$. The red precipitate was filtered, and the residue was co-evaporated 
thrice with menthol $(3 \times 10 \mathrm{ml})$ to afford the hydrobromide salt of naphthol-Ala (5.16 g, $16.5 \mathrm{mmol}, 94.5 \%)$.

MS(ESI): mass calculated for $\mathrm{C}_{13} \mathrm{H}_{13} \mathrm{~N}_{1} \mathrm{O}_{3}$ requires $m / z: 231.09$, found $[\mathrm{M}+1]^{+} \mathrm{m} / z$ 232.19; $[\mathrm{M}+\mathrm{Na}]^{+}$ $m / z 254.07$.

${ }^{1} \mathrm{H}-\mathrm{NMR}\left(500 \mathrm{MHz}, \mathrm{D}_{2} \mathrm{O}\right) \delta: 7.60(d, J=9.0 \mathrm{~Hz}, 1 \mathrm{H})$, $7.54(d, J=8.5 \mathrm{~Hz}, 1 \mathrm{H}), 7.50(\mathrm{~s}, 1 \mathrm{H}), 7.15(\mathrm{dd}, J=8.5$, $1.8 \mathrm{~Hz}, 1 \mathrm{H}), 7.03(d, J=2.4 \mathrm{~Hz}, 1 \mathrm{H}), 6.96(\mathrm{dd}, J=8.9$, $2.5 \mathrm{~Hz}, 1 \mathrm{H}), 4.20$ (dd, $J=7.6,5.7 \mathrm{~Hz}, 1 \mathrm{H}), 3.14$ (dd, $J=14.7,5.7 \mathrm{~Hz}, 1 \mathrm{H}), 3.15-3.08(\mathrm{~m}, 1 \mathrm{H})$.

${ }^{13} \mathrm{C}$-NMR $\left(126 \mathrm{MHz}, \mathrm{D}_{2} \mathrm{O}\right) \delta: 170.35,152.66,132.87$, $128.97,127.85,127.60,126.80,126.55,117.51,108.35$, $53.37,34.76$.

\section{Plasmids and cell lines used}

The plasmid pBK-lib-jw1 encodes a library of Methanococcus jannaschii tyrosyl tRNA synthetase (TyrRS) mutants randomized at residues Tyr32, Leu65, Phe108, Gln109, Asp158, and Leu162. Any one of Ile63, Ala67, His70, Try114, Ile159, and Val164 is also either mutated to Gly or kept unchanged. Plasmid pREP(2)/YC encodes MjtRNA $\mathrm{CUA}_{\mathrm{Cu}}^{\mathrm{Tyr}}$ the CAT gene with a TAG codon at residue 112, the GFP gene under the control of the T7 promoter, and a Tetr marker. Plasmid pLWJ17B3 encodes $M j$ tRNA ${ }_{\text {CUA }}^{\mathrm{Tyr}}$ under the control of the lpp promoter and $r r n C$ terminator, the barnase gene (with three amber codons at residues 2, 44, and 65) under the control of the ara promoter, and an Ampr marker. Plasmid pBAD/JYAMB-4TAG encodes the myoglobin gene of mutant sperm whale with an arabinose promoter and $r r n B$ terminator, $M j$ tRNA $A_{C U A}^{\mathrm{Tyr}}$ with an $l p p$ promoter and $r r n C$ terminator, and a tetracycline resistance marker.

\section{Genetic selection of the mutant synthetase specific for naphthol-Ala}

pBK-lib-jw1 consisting of $2 \times 10^{9}$ TyrRS-independent clones was constructed using standard PCR methods. E. coli strain DH10B harboring the pREP(2)/YC plasmid was used as the host strain for positive selection. Cells were transformed with the pBK-lib5 library, recovered in SOC for $1 \mathrm{~h}$, and washed twice with glycerol minimal media with leucine (GMML) before plating on GMMLagar plates supplemented with $50 \mu \mathrm{g} / \mathrm{mL}$ kanamycin, $60 \mu \mathrm{g} / \mathrm{mL}$ chloramphenicol, $15 \mu \mathrm{g} / \mathrm{mL}$ tetracycline, and $1 \mathrm{mmol} / \mathrm{L}$ naphthol-Ala. The plates were incubated at $37{ }^{\circ} \mathrm{C}$ for $60 \mathrm{~h}$, the surviving cells were scraped, and the plasmid DNA was extracted and purified by gel electrophoresis. pBK-lib-jw1 DNA was then transformed into electro-competent cells harboring the negative selection plasmid pLWJ17B3, recovered for $1 \mathrm{~h}$ in SOC, and then plated on LB-agar plates containing $0.2 \%$ arabinose, $50 \mu \mathrm{g} / \mathrm{mL}$ ampicillin, and $50 \mu \mathrm{g} / \mathrm{mL}$ kanamycin. The plates were incubated at $37{ }^{\circ} \mathrm{C}$ for $8-12 \mathrm{~h}$, followed by a similar extraction of pBK-lib5 DNA from the surviving clones. The library was then carried through a subsequent round of positive selection, followed by negative selection and a final round of positive selection (with $70 \mu \mathrm{g} / \mathrm{mL}$ chloramphenicol). At this stage, 96 individual clones were selected, suspended in $50 \mathrm{ml}$ of GMML in a 96-well plate, and replica-spotted on two sets of GMML plates. A set of GMML-agar plates was supplemented with $15 \mu \mathrm{g} / \mathrm{mL}$ tetracycline; $50 \mu \mathrm{g} / \mathrm{mL}$ kanamycin; $60,80,100$ or $120 \mu \mathrm{g} / \mathrm{mL}$ chloramphenicol; and $1 \mathrm{mmol} / \mathrm{L}$ naphtholAla. Another set of identical plates without naphthol-Ala was produced with $0,20,40$, or $60 \mu \mathrm{g} / \mathrm{mL}$ chloramphenicol. After $60 \mathrm{~h}$ of incubation at $37^{\circ} \mathrm{C}$, one clone was found to survive at $100 \mu \mathrm{g} / \mathrm{mL}$ chloramphenicol in the presence of $0.5 \mathrm{mmol} / \mathrm{L}$ naphthol-Ala, and at $20 \mu \mathrm{g} / \mathrm{mL}$ chloramphenicol in the absence naphthol-Ala.

\section{Generation of mutants and site-directed mutagenesis analysis}

Plasmid pET22b containing cpsfGFP was used to generate mutant cpsfGFPY66amber. Mutagenesis was confirmed through DNA sequencing analysis.

\section{Expression and purification of cpsfGFP-66- naphthol-Ala}

To express mutant cpsfGFP, plasmid pET22b-cpsfGFP66-TAG was co-transformed with pEOVL-naphthol-Ala synthetase (RS) into BL21(DE3) E. coli cells. Cells were amplified in $5 \mathrm{ml}$ of LB media supplemented with $50 \mu \mathrm{g} / \mathrm{mL}$ ampicillin and $30 \mu \mathrm{g} / \mathrm{mL}$ chloramphenicol. A starter culture $(1 \mathrm{ml})$ was used to inoculate $100 \mathrm{ml}$ of liquid LB supplemented with appropriate antibiotics and $0.5 \mathrm{mmol} / \mathrm{L}$ naphthol-Ala. The cells were then grown at $37{ }^{\circ} \mathrm{C}$ to an $O D_{600}$ of 1.1 , and protein production was induced by the addition of $0.2 \%$ arabinose and $1 \mathrm{mmol} / \mathrm{L}$ IPTG. After $12 \mathrm{~h}$, the cells were harvested by centrifugation and then sonicated. The supernatant was collected and incubated with Ni-NTA agarose beads for $1 \mathrm{~h}$ at $4{ }^{\circ} \mathrm{C}$, filtered, and washed with wash buffer containing $50 \mathrm{mmol} / \mathrm{L}$ HEPES (pH 7.5), $500 \mathrm{mmol} / \mathrm{L} \mathrm{NaCl}$, and $20 \mathrm{mmol} / \mathrm{L}$ imidazole. The protein was eluted with the wash buffer containing $50 \mathrm{mmol} / \mathrm{L}$ HEPES, pH 7.5, $500 \mathrm{mmol} / \mathrm{L} \mathrm{NaCl}$, and $250 \mathrm{mmol} / \mathrm{L}$ imidazole. cpsfGFP-66-naphthol-Ala was loaded onto a MonoQ column and eluted with a $\mathrm{NaCl}$ gradient. cpsfGFP-66-naphthol-Ala was further purified by a Superdex 75 column (GE Healthcare) in buffer 
containing $20 \mathrm{mmol} / \mathrm{L}$ HEPES-NaOH (pH 7.5) and concentrated to $16 \mathrm{mg} / \mathrm{mL}$. Protein concentration was quantified using the Bradford assay.

\section{Structural determination of cpsfGFP-66- Naphthol-Ala}

Crystallization of cpsfGFP-66-naphthol-Ala was achieved through vapor diffusion against $100 \mathrm{mmol} / \mathrm{L}$ Tris $(\mathrm{pH}$ 6.0 ) and $20 \%$ PEG3K at $4{ }^{\circ} \mathrm{C}$. The crystals appeared within $3 \mathrm{~d}$ and were flash-frozen in liquid nitrogen. Diffraction data were collected at a wavelength of $0.979 \AA$ at BL17U of the Shanghai Synchrotron Radiation Facility with a Q315CCD detector. Data processing and reduction were carried out by using the HKL2000 package. The initial structure of cpsfGFP-66-naphtholAla was determined by molecular replacement with Molrep from the CCP4 suite by using the atomic coordinates of wild-type sfGFP (PDB code: 2B3P) as the search model. Molecular replacement solutions were modified and refined with alternative cycles of manual refitting. Structural refinement was carried out using PHENIX. During refinement, the Coot tool in the CCP4 program suite was used for the model building, ligand and water location, and real-space refinement of side chains and zones. The final structure of cpsfGFP-66naphthol-Ala was checked for geometrical correctness with PROCHECK. The collected data and structural refinement statistics are summarized in Table 5. Cartoons and other protein structure representations were generated using PyMOL (http://www.pymol.org), and the atomic coordinates and structure factors were deposited in Protein Data Bank.

\section{UV spectra of cpsfGFP-66-Naphthol-Ala}

$7 \mu \mathrm{mol} / \mathrm{L}$ solution of purified mutant cpsfGFP-66naphthol-Ala protein was added to different NaiP/citric acid buffers of various $\mathrm{pH}$, and their UV-Vis spectra were recorded using a quartz cuvette $(100 \mu \mathrm{l}, 1 \mathrm{~cm}$ path) at room temperature with Agilent 8453 UV-visible spectrophotometer.

\section{Excitation and emission spectra of cpsfGFP-66- naphthol-Ala}

The fluorescence spectra of a $2 \mu \mathrm{mol} / \mathrm{L}$ solution of purified mutant cpsfGFP-66-Naphthol-Ala protein were obtained using a Thermo Varioskan Flash instrument equipped with SkanIt 2.4.3 RE software.

Table 5 Summary of data-collection and refinement statistics

\begin{tabular}{|c|c|}
\hline Space group & $P 2_{1}$ \\
\hline Unit-cell parameters $(\AA)$ & $a=46.8, b=49.3, c=49.2$ \\
\hline Resolution range $(\AA)$ & $39.1-1.25(1.29-1.25)$ \\
\hline Number of unique reflections & 58,574 \\
\hline Data completeness (\%) & $99.7(100)$ \\
\hline$\langle I\rangle /\langle\sigma(I)\rangle$ & $17.7(2.8)$ \\
\hline$R_{\text {merge }}(\%)^{\mathrm{a}}$ & $6.4(42.5)$ \\
\hline$R$-factor $/ R_{\text {free }}(\%)^{\mathrm{b}}$ & $19.4 / 21.9$ \\
\hline r.m.s.d. bond length $(\AA ̊)$ & 0.007 \\
\hline r.m.s.d. bond angles $\left({ }^{\circ}\right)$ & 1.276 \\
\hline Number of atoms modeled & 2102 \\
\hline Number of water molecules & 215 \\
\hline Mean $B$ factor $\left(\AA^{2}\right)$ & 16.2 \\
\hline Protein main-chain atoms & 14.4 \\
\hline Protein side-chain atoms & 16.1 \\
\hline Water molecules & 25.3 \\
\hline \multicolumn{2}{|l|}{ Ramachandran plot statistics } \\
\hline Residues in most favored region (\%) & 96.0 \\
\hline Residues in additional allowed region (\%) & 3.6 \\
\hline Residues in disallowed region (\%) & 0.4 \\
\hline
\end{tabular}

${ }^{\mathrm{a}} R_{\text {merge }}=\sum_{h k l} \sum_{i}|I(h k l)-\langle I(h k l)\rangle| / \sum_{h k l} \sum_{i} \mathrm{I}(h k l)$, where $\langle I(h k l)\rangle$ is the main value of $I(h k l)$

${ }^{\mathrm{b}} R$-factor $=\sum|| F_{o b s}|-| F_{c a l c}|| / \sum\left|F_{o b s}\right|$, where $F_{\text {obs }}$ and $F_{\text {calc }}$ are the observed and calculated structure factors, respectively

The free $R$ factor was calculated using $5 \%$ of the reflections omitted from the refinement. Numbers in parentheses represent the value of the highest resolution shell 


\section{Circular dichroism (CD) experiments}

$10 \mu \mathrm{mol} / \mathrm{L}$ solution of purified mutant cpsfGFP-66naphthol-Ala in $10 \mathrm{mmol} / \mathrm{L} \mathrm{NaiP} /$ citric acid buffer at various $\mathrm{pH}$ was transferred into a quartz cuvette (200 $\mu \mathrm{l}, 1 \mathrm{~cm}$ path), and spectra were obtained at room temperature by using a CD spectrometer (Chirascan Plus). Scanning was done thrice at a scan speed of $120 \mathrm{~nm} / \mathrm{min}$ and 1-nm intervals. Data smoothing was not performed.

\section{Confocal and fluorescence microscopy}

Pre- and post-photoconversion images were obtained from a thin $(\sim 10 \mu \mathrm{m})$ layer of $E$. coli cells expressing the target protein in MOPS-citrate buffer (pH 5) using 405-nm excitation delivered using a 1.2-NA objective to the region within the circle in bleaching mode (Red channel: 580-645 nm; Red channel: 655-755 nm; scale bar: $50 \mu \mathrm{m}$ ). Photoconversion was performed at $37 \%$ laser power and 30 iterations at $111 \mathrm{~ms}$. All observations were obtained by confocal and fluorescence microscopy (FV1000, Olympus).

For photostability determination, samples were obtained by overnight induction of cpsfGFP-66-naphtholAla and eqFP650 expressed in the BL21 strain. Bacterial solution (1 ml) was centrifuged for $4 \mathrm{~min}$ at $4000 \mathrm{r} / \mathrm{min}$, and the pellet obtained was washed thrice with PBS buffer and suspended in $1 \mathrm{ml}$ of PBS buffer. Thereafter, $100 \mu \mathrm{l}$ of this solution was placed on a glass that had been preprocessed with polylysine. After $30 \mathrm{~min}$ of incubation, the glass was washed with $300 \mu$ of PBS buffer for microscopic observation. The samples were photobleached by $30 \mathrm{~W} / \mathrm{cm}^{2}$ of $561 \mathrm{~nm}$ light, and the time was recorded. All observations were obtained using an Olympus 71 microscopy.

Acknowledgements This work was financially supported in part by Grants from the National Natural Science Foundation of China (21750003, 91527302, U1632133, 31628004, 21473237, and 31628004); the National Key Research and Development Program of China (2017YFA0503704 and 2016YFA0501502); and the Key Research Program of Frontier Sciences, CAS (QYZDB-SSWSMC032). We thank S.S. Zang and X.H. Liu for their help with the NMR spectral analysis; Z. Xie for helping with the protein mass spectral analysis; J.H. Li for helping with the CD experiments; A.Y. Liu for helping with the optical microscopy experiments; and Y. Teng for helping with the confocal and fluorescence microscopy experiments.

\section{Compliance with Ethical Standards}

Conflict of interest Li Wang, Xian Chen, Xuzhen Guo, Jiasong Li, Qi Liu, Fuying Kang, Xudong Wang, Cheng Hu, Haiping Liu, Weimin Gong, Wei Zhuang, Xiaohong Liu, and Jiangyun Wang declare that they have no conflicts of interest.
Human and animal rights and informed consent This article does not contain any studies with human or animal subjects performed by the any of the authors.

Open Access This article is distributed under the terms of the Creative Commons Attribution 4.0 International License (http:// creativecommons.org/licenses/by/4.0/), which permits unrestricted use, distribution, and reproduction in any medium, provided you give appropriate credit to the original author(s) and the source, provide a link to the Creative Commons license, and indicate if changes were made.

\section{References}

Bogdanov AM, Mishin AS, Yampolsky IV, Belousov VV, Chudakov DM, Subach FV, Verkhusha VV, Lukyanov S, Lukyanov KA (2009) Green fluorescent proteins are light-induced electron donors. Nat Chem Biol 5:459-461

Chang H, Zhang MS, Ji W, Chen JJ, Zhang YD, Liu B, Lu JZ, Zhang JL, Xu PY, Xu T (2012) A unique series of reversibly switchable fluorescent proteins with beneficial properties for various applications. Proc Natl Acad Sci USA 109:4455-4460

Chen S, Tsao ML (2013) Genetic incorporation of a 2-naphthol group into proteins for site-specific azo coupling. Bioconjugate Chem 24:1645-1649

Chica RA, Moore MM, Allen BD, Mayo SL (2010) Generation of longer emission wavelength red fluorescent proteins using computationally designed libraries. Proc Natl Acad Sci USA 107:20257-20262

Chudakov DM, Matz MV, Lukyanov S, Lukyanov KA (2010) Fluorescent proteins and their applications in imaging living cells and tissues. Physiol Rev 90:1103-1163

Elowitz MB, Surette MG, Wolf PE, Stock J, Leibler S (1997) Photoactivation turns green fluorescent protein red. Curr Biol 7:809-812

Enterina JR, Wu LS, Campbell RE (2015) Emerging fluorescent protein technologies. Curr Opin Chem Biol 27:10-17

Gaussian 16, Revision B.01, Frisch MJ, Trucks GW, Schlegel HB, Scuseria GE, Robb MA, Cheeseman JR, Scalmani G, Barone V, Petersson GA, Nakatsuji H, Li X, Caricato M, Marenich AV, Bloino J, Janesko BG, Gomperts R, Mennucci B, Hratchian HP, Ortiz JV, Izmaylov AF, Sonnenberg JL, Williams-Young D, Ding F, Lipparini F, Egidi F, Goings J, Peng B, Petrone A, Henderson T, Ranasinghe D, Zakrzewski VG, Gao J, Rega N, Zheng G, Liang W, Hada M, Ehara M, Toyota K, Fukuda R, Hasegawa J, Ishida M, Nakajima T, Honda Y, Kitao O, Nakai H, Vreven T, Throssell K, Montgomery JA Jr, Peralta JE, Ogliaro F, Bearpark MJ, Heyd JJ, Brothers EN, Kudin KN, Staroverov VN, Keith TA, Kobayashi R, Normand J, Raghavachari K, Rendell AP, Burant JC, Iyengar SS, Tomasi J, Cossi M, Millam JM, Klene M, Adamo C, Cammi R, Ochterski JW, Martin RL, Morokuma K, Farkas O, Foresman JB, Fox DJ (2016) Gaussian, Inc., Wallingford CT

Grimm JB, English BP, Chen JJ, Slaughter JP, Zhang ZJ, Revyakin A, Patel R, Macklin JJ, Normanno D, Singer RH, Lionnet T, Lavis LD (2015) A general method to improve fluorophores for live-cell and single-molecule microscopy. Nat Methods 12:244

Laurent AD, Jacquemin D (2013) TD-DFT benchmarks: a review. Int J Quantum Chem 113:2019-2039

Liu XH, Li JS, Hu C, Zhou Q Zhang W, Hu MR, Zhou JZ, Wang JY (2013) Significant expansion of the fluorescent protein chromophore through the genetic incorporation of a metal-chelating unnatural amino acid. Angew Chem Int Edit 52:4805-4809 
Mou Y, Huang PS, Hsu FC, Huang SJ, Mayo SL (2015) Computational design and experimental verification of a symmetric protein homodimer. Proc Natl Acad Sci USA 112:10714-10719

Newman RH, Fosbrink MD, Zhang J (2011) Genetically encodable fluorescent biosensors for tracking signaling dynamics in living cells. Chem Rev 111:3614-3666

Nienhaus K, Nienhaus GU (2014) Fluorescent proteins for live-cell imaging with super-resolution. Chem Soc Rev 43:1088-1106

Niu W, Guo JT (2013) Expanding the chemistry of fluorescent protein biosensors through genetic incorporation of unnatural amino acids. Mol BioSyst 9:2961-2970

Saha R, Verma PK, Rakshit S, Saha S, Mayor S, Pal SK (2013) Light driven ultrafast electron transfer in oxidative redding of Green Fluorescent Proteins. Sci Rep 3:1580

Sengupta P, van Engelenburg SB, Lippincott-Schwartz J (2014) Superresolution imaging of biological systems using photoactivated localization microscopy. Chem Rev 114:31893202
Shaner NC, Patterson GH, Davidson MW (2011) Advances in fluorescent protein technology (vol 120, pg 4247, 2007). J Cell Sci 124:2321

Shcherbo D, Shemiakina II, Ryabova AV, Luker KE, Schmidt BT, Souslova EA, Gorodnicheva TV, Strukova L, Shidlovskiy KM, Britanova OV, Zaraisky AG, Lukyanov KA, Loschenov VB, Luker GD, Chudakov DM (2010) Near-infrared fluorescent proteins. Nat Methods 7:827-U1520

Shu XK, Royant A, Lin MZ, Aguilera TA, Lev-Ram V, Steinbach PA, Tsien RY (2009) Mammalian expression of infrared fluorescent proteins engineered from a bacterial phytochrome. Science 324:804-807

Subach FV, Verkhusha VV (2012) Chromophore transformations in red fluorescent proteins. Chem Rev 112:4308-4327

Subach FV, Piatkevich KD, Verkhusha VV (2011) Directed molecular evolution to design advanced red fluorescent proteins. Nat Methods 8:1019-1026 